\title{
Numbers and space: Associations and dissociations
}

\author{
Merav Ben Nathan \\ Tel-Aviv University, Tel-Aviv, Israel \\ Samuel Shaki \\ Ariel University Center of Samaria, Ariel, Israel \\ AND \\ Moti Salti And Daniel Algom \\ Tel-Aviv University, Tel-Aviv, Israel
}

\begin{abstract}
A cornerstone of contemporary research in numerical cognition is the surprising link found between numbers and space. In particular, people react faster and more accurately to small numbers with a left-hand key and to large numbers with a right-hand key. Because this contingency is found in a variety of tasks, it has been taken to support the automatic activation of magnitude as well as the notion of a mental number line arranged from left to right. The present study challenges the presence of a link between left-right location, on the one hand, and small-large number, on the other hand. We show that a link exists between space and relative magnitude, a relationship that might or might not be unique to numbers.
\end{abstract}

Is 7 odd or even? The time it takes people to answer this simple question depends on the side of the response key used to indicate parity. People respond faster to 7 and to other large (single) numbers with a right-hand key but they respond faster to 3 and to other small numbers with a lefthand key. The presence of this spatial-numerical association of response codes (SNARC) has given currency to the idea that numerical magnitude is activated in an automatic manner whenever a numeral is presented for any purpose (note that retrieval of numerical magnitude is not strictly required for parity decision). In this study, we challenge the idea of a unique, automatic link between numbers and space and argue instead that an automatically activated link exists between relative (numerical) magnitude and spatial location. We first show that the widely accepted idea of an association between a particular number and a specific spatial location is based on a common confound between absolute and relative magnitude in existing SNARC studies. We then show that removing the confound results in the collapse of the traditional number-based SNARC effect but not of a relative-magnitude-based SNARC effect.

The canonical experimental setup for assaying the SNARC effect entails manual performance of a task with numerals under alternative regimes of left-right key assignment. Under such conditions, the SNARC effect has been reported in a gamut of tasks-from parity decision (Dehaene, Bossini, \& Giraux, 1993) to samedifferent judgments (Dehaene \& Akhavein, 1995; but see Wood, Nuerk, \& Willmes, 2006) to detecting the presence of a phoneme in the name of the digit (Fias, Brys- baert, Geypens, \& d'Ydewalle, 1996) to bisection of lines composed of digits (Fischer, 2001) to finger-pointing the digit's location (Fischer, 2003) to judgments of numerical magnitude themselves (Gevers, Verguts, Reynvoet, Caessens, \& Fias, 2006). Apart from the last measure, what is remarkable about these tasks is that none strictly requires retrieval of numerical magnitude for successful performance. However, in virtually all SNARC studies, the two properties of number, absolute and relative magnitude, were inextricably yoked. Is it absolute magnitude or relative magnitude that is associated with location in space?

The difference between magnitude and relative magnitude (of, say, natural numbers) is subtle yet crucial for an understanding of the SNARC effect. The former notion is closely related to cardinal numbers, whereas the latter is closely related to ordinal numbers. The number 8 denotes a certain magnitude or numerosity (the first sense), but it also conveys the idea that 8 is larger than 5 or that it is smaller than 9 (the second sense). These two senses of number are confounded in existing research. Consider testing the SNARC effect with judgments of magnitude themselves (e.g., Gevers et al., 2006). In one block, the observer responds with the left-hand key when the presented number is smaller than 5 (the typical standard) and with the right-hand key when the number is larger than 5; in a second block, key assignment reverses. Note that within a block, the number 8 is always large and always maps onto the same fixed response key. Consequently, it is impossible to know whether the observer reacts to the cardinal arithmetic property of the number 8 or to the relatively large magnitude carried by

M. Ben Nathan, merav20@gmail.com 
8 (i.e., to the relation that 8 is larger than 5). In the present study, we disentangled the two properties and discovered that it is solely relative magnitude that is automatically retrieved and associated with spatial location.

The results of an experiment in the original study of the SNARC effect by Dehaene et al. (1993) already cast doubts on the idea that numbers per se are associated with locations in space. These authors generated a reversal of the SNARC effect by presenting common numbers in two different ranges of magnitude. People responded to the numbers 4 and 5 faster with a right-hand key in a context in which they appeared in the interval $0-5$ (when they were the largest numbers in that range). However, people responded to the same numbers faster with a left-hand key in a context in which they appeared in the interval 4-9 (when they were the smallest numbers). These results (see also, Fias et al., 1996) demonstrate that the relationship between a particular number and a particular locationthe SNARC effect-is dependent on context. In this study, we show that the bond with space is relativistic in an instantaneous fashion and hence is context independent.

Nevertheless, the confound between absolute and relative magnitude is present even in the aforementioned study by Dehaene et al. (1993). Within an experimental block, the number 4 was always large or always small and was mapped onto the same fixed response key. As a result, one cannot know whether the participants responded to the cardinal property of 4 (denoting a large numerosity in the set $0-5$ used) or to the ordinal property by which 4 was larger than the majority of the numbers in this set. It is also clear that the participants needed a time-consuming learning process by which the context was established. In contrast, we show that numbers are relative instantaneously, and it is this relativistic component that is associated with space. ${ }^{1}$

In the present study, we introduced a new experimental tactic that enabled the dissociation of the two components. The task for the participant was magnitude judgmentthat is, to decide, while timed, whether the single number presented for view was larger or smaller than a standard number. In one condition, the standard was fixed at 5. This is the traditional setup used in SNARC experiments with magnitude (e.g., Gevers et al., 2006; Ito \& Hatta, 2004). However, in our novel condition, the standard was not fixed, but changed from trial to trial in an unpredictable fashion. In this roving standard condition, the stimulus 8 was large if the momentary standard was 7 , but this same stimulus in the same block was small if the momentary standard was 9 . Therefore, in the roving standard condition, we pried apart the bond between relative and absolute magnitude and hence also violated the fixed mapping between number and response key within a block. Within the current experimental block, the number 8 was large and small an equal number of times. Inevitably, the number 8 was no longer associated with a constant response key within the block.

If the SNARC effect depends on numerical magnitude, then responses to 8 would be faster with the right-hand key than with the left-hand key throughout the trials in the block (with a roving standard). Because 8 is always a large (single) number, the right-hand response key would be preferred regardless of the value of the momentary standard.
The commonly observed SNARC function is expected to emerge in the roving standard condition, too, under the hypothesis of an absolute magnitude effect. However, if the underlying association is not between number and space, but rather between relative magnitude and space, then responses to the same numeral in the same block will vary depending on the relative magnitude that it carries. Thus, within the same block, the responses to the same stimulus, 8 , will be faster with the left-hand key when 8 carries a small relative magnitude (i.e., smaller than the momentary standard of 9), but will be faster with the right-hand key if it carries a relatively large magnitude (i.e., larger than the momentary standard 7). Under the hypothesis of a relative magnitude effect, one expects to find two parallel curves along the vertical axis, one for the numbers when they were relatively small (at the top) and one for the same numbers when they were relatively large (at the bottom).

Applying the novel roving standard procedure, we were able to disentangle the two components and show that only relative magnitude counts. These results raise the intriguing possibility that the SNARC effect itself documents an incidental association between numbers and space brought about by the trivial fact that numbers are carriers of relative magnitude.

\section{The Present Study: \\ Fixed Versus Roving Standard}

We tested the SNARC effect for judgments of magnitude under two different conditions. In the first condition, we followed accepted practice: The standard was fixed at 5 and the participant's task was to decide whether the presented number was larger or smaller than 5 . In the second condition, we deviated from accepted practice: The standard was not fixed but varied from trial to trial in an unpredictable fashion. Both conditions entailed two blocks. In one block, the participant indicated large magnitude (i.e., number greater than standard) by pressing a left-hand key and small magnitude by pressing a righthand key. In another block, the participants responded through the reverse key assignment. The SNARC effect is present if the responses to small numbers are faster with the left-hand key than with the right-hand key and, similarly, if the responses to large numbers are faster with the right-hand key than with the left-hand key. However, if the responses depend on relative numerical magnitude, then the left-right asymmetry would be determined by the momentary standard in the second condition.

\section{METHOD}

\section{Participants}

Eighteen Carleton University undergraduate students volunteered to participate in the study. All of them had normal or corrected-tonormal vision and were unaware of the purpose of the study.

\section{Stimuli and Apparatus}

The stimulus set comprised the digits from 1-9. Each number extended $0.6 \mathrm{~cm}$ in height and $0.3 \mathrm{~cm}$ in width and appeared in black at the center of the screen. The stimuli that served as standard numbers in the roving standard condition extended $0.4 \mathrm{~cm}$ in height and $0.2 \mathrm{~cm}$ in width and appeared at the top, so that they were completely distinctive, perceptually, from the probe numbers. The presentation 
of the stimuli, event sequencing and timing, and the recording of the responses were governed by a Pentium III computer running under SuperLab control.

The fixed standard condition. Because the (fixed) standard was 5 , it was not used as a test stimulus. The remaining eight stimuli were presented 20 times each in random order, resulting in a block of 160 trials. The block was preceded by 10 trials for practice (unbeknownst to the participants). The order of presentation was random and different for each participant. The participant's task was to decide, while timed, whether the presented number was smaller or larger than 5 . The participant responded by pressing the appropriate lateralized key. In two blocks, the assignments of the keys for large and small responses were reversed.

The roving standard condition. The probe digits were all of the numbers from 2-8. The extreme numbers of 1 and 9 were not used because they could not be rendered small and large by any momentary standard (of a single digit). Each probe number was preceded by a standard number, which varied from trial to trial. The probe number followed the standard after a 500 -msec interval. The following 14 standard-stimulus pairs were presented (the first number is the standard): $(1,2),(5,2),(2,3),(4,3),(3,4),(8,4),(2,5),(7,5)$, $(4,6),(9,6),(5,7),(8,7),(6,8),(9,8)$. Note that in this arrangement each stimulus number was small and large an equal number of times. Each pair was presented 11 times, making for a total of 154 trials in a block. The participant's task was to decide, while timed, whether the number was larger or smaller than the standard number at the top. The participant responded by pressing the appropriate lateralized key. In two blocks, the assignments of the keys for large and small responses were reversed.

\section{Procedure}

The participants were tested individually in a dimly lit room. They were seated approximately $60 \mathrm{~cm}$ from the center of the screen. The responses were made by pressing one of the marked keys (" $\mathrm{Z}$ " on the left, and "M" on the right). In one block of each condition, the participants indicated "smaller than standard" by pressing the lefthand key and "larger than standard" by pressing the right-hand key. In the other block, they responded via the reverse key assignment. The order of the blocks within each condition was counterbalanced between participants. The order of conditions was also strictly counterbalanced. The stimuli were response terminated, and a new stimulus was presented after a 500-msec interval. Participants were encouraged to respond quickly, but accurately.

\section{RESULTS}

\section{The Fixed Standard Condition}

Figure 1 shows the difference in response time (RT) between the right- and the left-hand responses (dRT = right - left), plotted against numerical magnitude. The negative slope $(-34.803)$ of the regression line depicts the presence of the SNARC effect in this condition [intercept $\left.=165.654, r^{2}=.891 ; t(7)=7.006, p<.0001\right]$. For small numbers, the left-hand responses were faster than the right-hand responses, whereas for large numbers, the pattern reversed. The number $\times$ hand interaction in the overall ANOVA $[F(7,119)=5.564, p<.0001]$ documents once again the presence of an appreciable SNARC effect with a fixed standard. The simple main effect for number was reliable [larger number tended to produce shorter RTs; $F(7,119)=28.089, p<.0001]$ but the simple main effect for hand was not $[F(1,17)=2.03, p=.172]$.

In order to further assay the SNARC effect, we used Lorch and Myers's (1990) repeated measures regression analyses. For each participant, we derived the standardized beta regression coefficients with dRT as the depen-

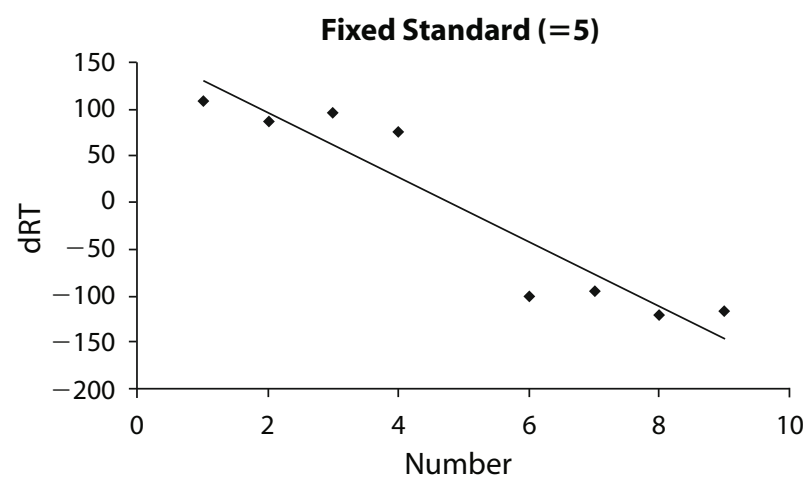

Figure 1. Fixed standard: The difference in mean response time (RT, in milliseconds) between the right- and the left-hand keys $(\mathrm{dRT}=$ right - left $)$, plotted against number. The negative slope of the dRT function documents the SNARC effect.

dent variable and numerical magnitude as the predictor. These weights $(M=-0.4263, S D=0.56149)$ differed significantly from zero $[t(17)=-3.221, p<.005]$. Of the 18 participants, the data of 14 displayed the typical steady negative slope. The pattern of Figure 1 thus documents the presence of a reliable SNARC effect for judgments of magnitude when using a fixed standard for comparison.

Visual inspection of Figure 1 also reveals a categorical component (with groups of points concentrated in the upper left for small numbers and in the lower right for large numbers). To test for the presence of this component, we conducted another regression analysis using a categorical predictor instead of the linear predictor. A much larger negative slope $(-200.67)$ of the regression line was supported by an improved goodness of fit [intercept $\left.=292.653, r^{2}=.988 ; t(7)=-6.8, p<.0001\right]$. Our results are thus consistent with the presence of a categorical component in judgments of magnitude much like that uncovered by Gevers et al. (2006) and more recently by Fitousi, Shaki, and Algom (2009). Nevertheless, given (1) the reasonably good linear fit (explaining $90 \%$ of the variance), (2) the presence of this and even better values of fit with 14 of the individual participants), and (3) the need to consider noncategorical data in the other condition, we used the parameters of the linear fit for further analyses and comparisons. We sought to answer the following question: Does the SNARC effect obtain when the standard is not fixed, but rather changes from trial to trial in a random fashion?

\section{The Roving Standard Condition}

Figure 2 shows the parallel results in the condition with a roving standard. As in Figure 1, the difference, dRT $=$ $\mathrm{RT}$ (right) - RT(left), is plotted on the ordinate against the number on the abscissa. Two curves result. The one on the top depicts the dRT for each of the numbers when it was smaller than the momentary standard. The curve at the bottom depicts the dRT for the same numbers when they were larger than their momentary standards. The two curves are separated along the vertical dimension. The separation is very large, so that virtually none of the respective ranges of values overlap $[t(6)=16.9, p<.0001]$. 


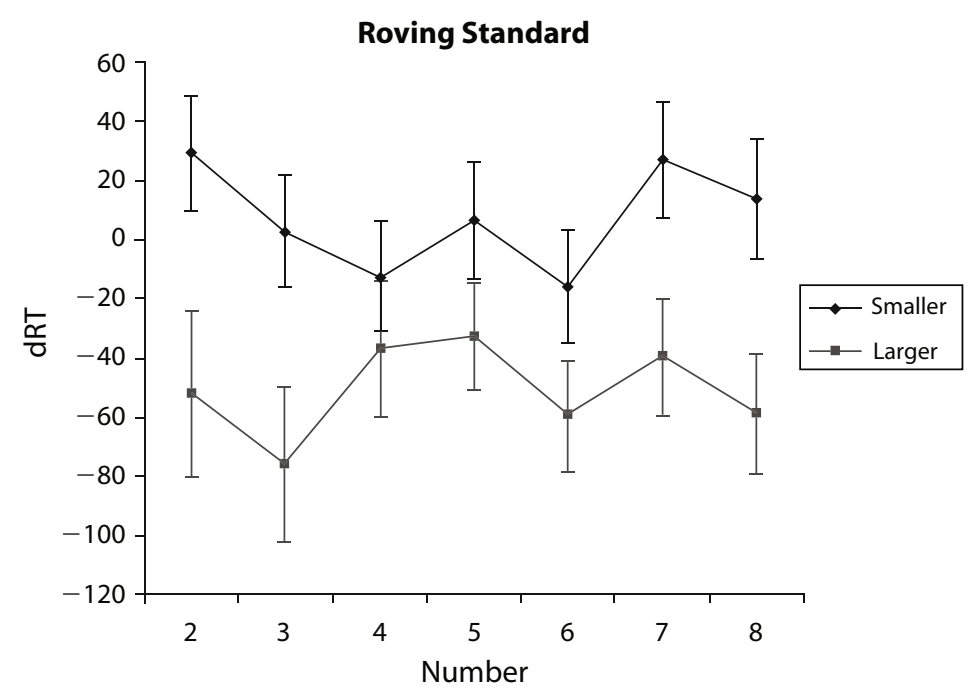

Figure 2. Roving standard: The difference in mean response time (RT, in milliseconds) between the right- and the left-hand keys $(\mathrm{dRT}=$ right - left $)$, plotted against number. The curve at the top depicts the dRT for all cases in which the numbers were smaller than their momentary standards. The curve at the bottom depicts dRT for the same numbers when they were larger than their momentary standards.

The results summarized in Figure 2 tell a startling story. When a given number was larger than the momentary standard, the responses were faster by the right-hand key. However, when the same number was smaller than the momentary standard, the responses were faster by the left-hand key. Take the number 8 as an example. When the standard was 9 , the responses to 8 were faster with the left-hand key than with the right-hand key. However, when the standard was 6 , the responses to 8 were faster with the right-hand key than with the left-hand key.

A corollary of these results is the collapse of the standard SNARC effect in this condition with a roving standard. Indeed, the lack of a number $\times$ hand interaction $(F<1)$ confirmed the complete absence of the standard SNARC effect from judgments of magnitude with a roving standard. In an additional analysis (recommended by Schwarz $\&$ Keus, 2004), we divided the numbers into a small absolute magnitude group and a large absolute magnitude group. Entering these categories for the number variable into a parallel ANOVA resulted again in an absence of a number $\times$ hand interaction $[F(1,17)=0.040, p=.844]$.

In yet another analysis, we calculated for each participant the standardized beta regression coefficients with $\mathrm{dRT}$ as the dependent variable and number as the predictor. The weights $(M=0.1063, S D=0.49136)$ did not differ from zero $[t(17)=0.918, p=.371]$, reinforcing the disappearance of the routine SNARC effect from the roving standard condition.

In a final analysis aimed at ruling out conclusively any role for absolute magnitude in generating the SNARC effect, we performed a multiple regression (on data pooled over the individual participants) with $\mathrm{dRT}$ as the dependent variable and (absolute) number and relative magnitude (vis-à-vis the momentary standard) as the predictors. The results showed that relative magnitude was the sole predictor of dRT [for relative magnitude, $t(1)=-5.1$, $p<.0001$; for number, $t(6)=0.577, p=.575]$. Relative magnitude explained $70 \%$ of the variance. This outcome (along with those of the other analyses) shows that relative magnitude is the sole generator of the standard SNARC effect. This last conclusion invites a new, alternative interpretation of the SNARC effect.

\section{Overall Comparison Across Conditions}

Direct comparison between the two conditions supports the conclusion that the traditional SNARC effect is based solely on relative numerical information. An overall ANOVA on RT with standard (fixed, roving), hand (left, right), and number [small, 1-4 (first condition) and 2-4 (second condition); large, 6-9 (first condition) and 6-8 (second condition)] as main factors yielded reliable threeway interaction $[F(1,17)=4.97, p<.05]$. The presence of this interaction means that the type of standard made a difference: The traditional SNARC effect (hand $\times$ number) was present with a fixed standard but absent with a roving standard. The latter is the critical result because the second condition provided the conclusive test for deciding between the role of relative versus absolute magnitude. The outcome of the first condition (as well as that of the great bulk of existing SNARC studies) cannot provide a resolution because relative and absolute magnitude are confounded in that design (e.g., the number 8 is large in an absolute and in a relative sense).

We reported earlier that the dRT number regression coefficients differed from zero only in the first condition (documenting the presence of the SNARC effect in that condition). A direct comparison of the beta coefficients across the two conditions reinforced the presence of the SNARC effect in the first condition but its absence from the second $[t(17)=-2.88, p=.01]$. 
In sum, the omnibus analyses show decisively that the SNARC effect rests on the ordinal property of numbers and that this property is activated in an instantaneous manner.

\section{DISCUSSION}

Each (natural) number at once denotes a cardinal and an ordinal value. There is some controversy in number theory as to the primacy of these properties (e.g., Frege, 1884; Russell, 1919), although both form part of any (axiomatic) definition of number (Dedekind, 1901/1963; Peano, 1889/1967). The results of this study reveal, quite surprisingly, that it is the ordinal property of number that counts when attempting to explain the wealth of numberspace findings gathered under the rubric of the SNARC effect. This result invites reexamination of the origin of the SNARC effect.

Earlier research (Dehaene at el., 1993; Fias et al., 1996; Fischer, 2006; Fischer \& Coeman, 2005) has already suggested that the number-space association-the SNARC effect - is context dependent. The present results show that context and training are not essential for the relativity effect. The effect is instantaneous: What matters is the relative magnitude that a number happens to carry at any given moment. In other words, relativity is not a sideproduct of learning or an offbeat result obtained under special circumstances but rather is the true fundamental cause of the SNARC effect. If so, the effect might not be a numerical effect at all —only the incidental association of numbers with magnitude makes it look like one.

To a first approximation, our results seem to be consistent with the binary-choice model of Proctor and Cho (2006) as well as with the computational model developed by Gevers et al. (2006; see also the earlier model by Verguts, Fias, \& Stevens, 2005, as well as the model by Keus \& Schwarz, 2005). The Gevers et al. model entails a dualroute architecture in which a numeral is automatically coded as large or small, regardless of the task at hand. It is this relativistic component that is associated with space. The second and relatively slow route is conditional on task instructions. For this strategic component, the model assumes that there is a connection, built up over lifetime experience, between small(ness) and left, on the one hand, and large(ness) and right, on the other hand. Notably, any "specific number is not coded as left or right" (Gevers et al., 2006, p. 41, emphasis added), nor are such connections needed to account for the SNARC effect. The response mapping of the second route may or may not be compatible with that of the first route (the only route that is associated with spatial codes). The response congruency or conflict thus produced is the root cause of the observed SNARC effect. We share with the Gevers et al. model the assumption about the absence of a genuine bond between number and space; extensive simulations are needed to see whether the present data are fully consistent with the predictions of the model.

\section{AUTHOR NOTE}

We thank three anonymous reviewers for valuable comments on an earlier version of this article. We also thank Bill Petrusic for offering the facilities of his laboratory as well as for helpful input. Correspondence concerning this article should be addressed to M. Ben Nathan, Department of Psychology, Tel-Aviv University, Ramat-Aviv 69978, Israel (e-mail: merav20@gmail.com).

\section{REFERENCES}

DEDEKIND, R. (1963). Essays on the theory of numbers. New York: Dover. (Original work published 1901)

Dehaene, S., \& AkHavein, R. (1995). Attention, automaticity, and levels of representation in number processing. Journal of Experimental Psychology: Learning, Memory, \& Cognition, 21, 314-326.

Dehaene, S., Bossini, S., \& Giraux, P. (1993). The mental representation of parity and number magnitude. Journal of Experimental Psychology: General, 122, 371-396.

Fias, W., Brysbaert, M., Geypens, F., \& D’Ydewalle, G. (1996). The importance of magnitude information in numerical processing: Evidence from the SNARC effect. Mathematical Cognition, 2, 95-110.

FISCHER, M. H. (2001). Number processing induces spatial performance biases. Neurology, 57, 822-826.

FISCHER, M. H. (2003). Spatial representation in number processing: Evidence from a pointing task. Visual Cognition, 10, 493-508.

Fischer, M. H. (2006). The future for SNARC could be stark. Cortex, 42, 1066-1068.

Fischer, M. H., \& Coeman, P. (2005, July). Moving the mental number line: Rapid effects of training. Poster session presented at the European Summer School on "Neuroscience of Number Processing," Erice, Italy.

Fitousi, D., Shaki, S., \& Algom, D. (2009). The role of parity, physical size, and magnitude in numerical cognition: The SNARC effect revisited. Attention, Perception, \& Psychophysics, 71, 143-155.

Frege, G. (1884). Die Grundlagen der Arithmetik. Jena: Hermann Pohle.

Gevers, W., Verguts, T., Reynvoet, B., Caessens, B., \& Fias, W. (2006). Numbers and space: A computational model of the SNARC effect. Journal of Experimental Psychology: Human Perception \& Performance, 32, 32-44.

Ito, Y., \& HatTa, T. (2004). Spatial structure of quantitative representation of numbers: Evidence from the SNARC effect. Memory \& Cognition, 32, 662-673.

Keus, I. M., \& Schwarz, W. (2005). Searching for the functional locus of the SNARC effect: Evidence for a response-related origin. Memory \& Cognition, 33, 681-695.

LORCH, R. F., JR., \& MYERS, J. L. (1990). Regression analysis of repeated measures data in cognition research. Journal of Experimental Psychology: Learning, Memory, \& Cognition, 16, 149-157.

Peano, G. (1967). The principles of arithmetic, presented by a new method. In J. van Heijenoort (Ed.), A source book in mathematical logic, 1879-1931 (pp. 83-97). Cambridge, MA: Harvard University Press. (Original work published 1889)

Proctor, R. W., \& Cho, Y. S. (2006). Polarity correspondence: A general principle for performance of speeded binary classification tasks. Psychological Bulletin, 132, 416-442.

RUSSELL, B. (1919). Introduction to mathematical philosophy. London: Allen \& Unwin.

SchWARz, W., \& Keus, I. M. (2004). Moving the eyes along the mental number line: Comparing SNARC effects with saccadic and manual responses. Perception \& Psychophysics, 66, 651-664.

Verguts, T., Fias, W., \& Stevens, M. (2005). A model of exact smallnumber representation. Psychonomic Bulletin \& Review, 12, 66-80.

Wood, G., Nuerk, H. C., \& Willmes, K. (2006). Crossed hands and the SNARC effect: A failure to replicate Dehaene, Bossini, and Giraux (1993). Cortex, 42, 1069-1079.

\section{NOTE}

1. Our predictions in this study apply most readily, of course, to SNARC experiments using magnitude judgment as the task. However, the same considerations apply in general to all SNARC experiments; all entail the confound, and it is always the relativistic component that generates the effect.

(Manuscript received June 25, 2008; revision accepted for publication December 13, 2008.) 